\title{
Reported disability in relation to observed activity limitation, grip strength and physical function in women and men with rheumatoid arthritis
}

Sidona-Valentina Bala ${ }^{1,2^{*}}$ (D), Maria L. E. Andersson ${ }^{3,4}$, Kristina Forslind ${ }^{3,5}$, Björn Svensson ${ }^{3}$ and Ingiäld Hafström ${ }^{6,7}$

\begin{abstract}
Background: The self-reported Health Assessment Questionnaire (HAQ) is specifically designed to assess disability in arthritic patients. In many studies women report higher functional disability than men. The reasons for this difference are suggested to be multifactorial. We therefore evaluated functional disability assessed by HAQ in women and men with rheumatoid arthritis (RA) in relation to observed disability, grip force and physical function.

Methods: Patients with RA, 51 women and 49 men, completed the HAQ on three occasions, some weeks apart. Between HAQ1 and HAQ2, all patients performed 17 of the 20 activities (7 domains) included in the HAQ under observation in a specially designed environment, the observed HAQ. During the same day, grip force, measured by GRIPPIT and physical function assessed by the SOFI (Signals of Functional Impairment) index were evaluated. Differences between groups were studied by the chi-square test, Mann-Whitney $U$ test and Wilcoxon Sign Rank test. Correlations were analysed by Spearman rank correlation. Comparisons between repeated measures were performed using Friedman's test.
\end{abstract}

Results: Median (IQR) total HAQ1 score was 0.50 (0.88) for women and 0.25 (0.84) for men, $p=0.038$, and the observed HAQ score (7 domains) 0.57 (0.9) for women and 0.43 (0.96) for men, $p=0.292$. The correlations between reported HAQ1 score (7 domains) and observed HAQ score were strong, $r=0.860, p<0.001$ in women, and $r=$ $0.820, p<0.001$ in men. For some activities the patients, both women and men, reported lower difficulty than that observed. Women had lower grip force than men, median (IQR), right and left 126 (84) Newton, versus 238 (146), $p<0.001$, and there was a negative correlation between grip force and most of the separate activities in HAQ in both genders. SOFI index was similar in women and men, median (IQR) 0 (3.0) versus 0 (2.0), $p=0.277$, with a moderate correlation to HAQ.

Conclusions: The results indicate that in well-treated patients with RA the correlations between reported and observed HAQ scores were strong, similarly in women and men. We found no evidence that the patient's opinion was dependent on unawareness of her/his own ability.

Keywords: Rheumatoid arthritis, Disability, HAQ, Mobility, Hand strength, Women, Men

\footnotetext{
* Correspondence: sidona-valentina.bala@skane.se

'Department of Health Sciences, Faculty of Medicine, Lund University, Lund, Sweden

2Department of Medicine, Section of Rheumatology, Helsingborg's Hospital, Helsingborgs lasarett, Olympiahuset plan 2, S-251 87 Helsingborg, Sweden Full list of author information is available at the end of the article
}

(c) The Author(s). 2021 Open Access This article is licensed under a Creative Commons Attribution 4.0 International License, which permits use, sharing, adaptation, distribution and reproduction in any medium or format, as long as you give appropriate credit to the original author(s) and the source, provide a link to the Creative Commons licence, and indicate if changes were made. The images or other third party material in this article are included in the article's Creative Commons licence, unless indicated otherwise in a credit line to the material. If material is not included in the article's Creative Commons licence and your intended use is not permitted by statutory regulation or exceeds the permitted use, you will need to obtain permission directly from the copyright holder. To view a copy of this licence, visit http://creativecommons.org/licenses/by/4.0/ The Creative Commons Public Domain Dedication waiver (http://creativecommons.org/publicdomain/zero/1.0/) applies to the data made available in this article, unless otherwise stated in a credit line to the data. 


\section{Background}

In the last decades, reports have emerged that women with rheumatoid arthritis (RA) have a more severe disease course than men [1-6]. In both genders, disease activity decreases over the first 8 years from disease onset but more in men, whereas functional disability, after an initial decrease, levels off at a higher level in women than in men $[7,8]$. After 5 and 8 years from disease onset, women have higher disease activity as measured by the Disease Activity Score 28 (DAS28) and higher functional disability measured by Health Assessment Questionnaire (HAQ) than men [7-9]. These differences between genders contrast with the fact that joint destruction in hands and feet was similar in women and men, both after 5 and 8 years from disease onset $[5,6]$. The reasons for these gender differences have not yet been identified. As to functional disability, potential causes may be that women's grip force is lower [10], that men overestimate their functional ability [5, 11], that men have better muscle strength [12] and /or that women report more pain [13-15]. Another reason may be that HAQ is not sufficiently developed with regard to possible gender differences and therefore cannot handle functional differences between the sexes. This is of great importance to be clarified as HAQ is the most widely used instrument to assess disability in studies of RA. Moreover, HAQ has proved to be a predictor of disability [16] and sustained remission in the course of RA [17], and also a decision support for need of multidisciplinary interventions [18]. Therefore, additional knowledge about the reasons for the differences between women's and men's subjective assessments of their functional disability is necessary.

The aims of the present study were to evaluate functional disability assessed by HAQ in women and men with RA in relation to observed disability, grip force and physical function.

\section{Methods \\ Design and setting}

This is a cross-sectional study performed in an outpatient rheumatology clinic located at a central hospital in Southern Sweden.

\section{Patients}

In all, 100 patients fulfilling the classification criteria for RA established by the American Rheumatism Association [19] were included in the study. The women and men were separately consecutively included with the aim to achieve about equal numbers. The patients were eligible for inclusion if they were 18-74 years of age, had a disease duration less than 15 years and a good understanding of the Swedish language. They were asked for participation in the study in connection with their regular visits to a nurse. Nine patients were not asked to participate - 4 were wheelchair users (3 women and 1 man) and 5 had undergone hip surgery (1 woman and 4 men) - as their functional limitations might have affected their ability to perform some of the activities in the study protocol. Fifteen patients (6 women and 9 men) declined participation because they did not have any opportunities to return to the clinic within 2 weeks according to stage 2 in the study protocol. All included patients completed the study.

\section{Assessments}

\section{Health assessment questionnaire (HAQ)}

Functional disability was assessed by the Swedish version of the HAQ [20]. The HAQ comprises of 20 questions covering 20 daily activities. These are divided in eight domains: dressing and grooming, rising, eating, walking, hygiene, reaching, gripping, and other activities. Each question is scored according to a four-point scale (0-3), and the highest scores from each domain are summed and divided by eight, to derive a total HAQ score, which ranges from 0 to 3 ( 3 = highest level of disability).

\section{Disease activity score 28 (DAS28)}

Disease activity was assessed by the composite index Disease Activity Score calculated in 28 joints (DAS28; range 0-9.4, best to worse) [21].

\section{Grip force}

Grip force was assessed in both hands by the electronic instrument GRIPPIT, which measures grip force in newtons $(\mathrm{N})$ [22]. Average values over a $10 \mathrm{~s}$ uninterrupted grip were recorded. Results are given for the separate hands as well as means for right and left hands.

\section{Physical function}

Physical function was assessed by the SOFI (Signals Of Functional Impairment) index which is a three parts measurement of hands (range 0-16), upper extremities/arms (range 0-12), and lower extremities/legs (range 0-16) [23]. The SOFI index ranges from 0 (best) to 44 (worst).

\section{Data collection procedure}

Data were collected at baseline (stage 1), within 2 weeks after baseline (stage 2), and within 3 weeks after stage 2 (stage 3).

\section{Stage 1}

At a regular visit to the nurse, all patients were asked to complete a HAQ, hereinafter referred to as HAQ1. Demographic data and their last DAS28 (values not older than 6 month) were also recorded. The patients were then planned for a new visit to the nurse within 2 weeks. 


\section{Stage 2}

At a second visit all patients performed 17 of the 20 activities included in the HAQ in a specially designed environment within the hospital in order to compare their subjective assessments with their observed functional abilities. These 17 activities corresponded to 7 of the 8 domains in the questionnaire. Exceptions were made for the activities in the first domain (hair washing and dressing, corresponding to questions 1 and 2 in the HAQ) and for one activity in the eighth domain (common activities, corresponding to the question "Can you handle your own household purchases?") because these activities were difficult to perform in hospital environment.

During this assessment procedure, which lasted for 45 $\min (\min )$ to $65 \min (\max )$, the patient was assisted by a nurse who observed and estimated the actual functional performance. The nurse documented her assessment in the HAQ questionnaire (hereinafter referred to as observed HAQ), without any feedback to the patient of how her/his performance was assessed. To facilitate and ensure consistency in the nurse's assessment, a standardized protocol which described the different levels of ability for the HAQ-scale's response categories "With some difficulty" and "With much difficulty" has been developed by using expert opinion (rheumatologists, occupational therapist, nurse and health care researchers) (Table 1). The protocol was tested on 4 patients, 2 men and 2 women, which did not result in any adjustments. According to the protocol the time factor was not considered as an assessment criterion.

After performing the observed activities, the patients were asked to complete the HAQ questionnaire once again (hereinafter referred to as HAQ2).

At this time point the occupational therapist assessed the patients' physical function in the upper and lower extremities by SOFI, and their grip force for both hands by GRIPPIT.

\section{Stage 3}

One to 3 weeks after stage 2 , the patients were asked to complete a final HAQ questionnaire (HAQ3). The HAQ3 questionnaire was marked with date and sent to the patients by post together with a response envelope. Patients who did not return the questionnaire within 2 weeks were reminded by telephone.

\section{Statistical analysis}

Demographics and disease activity data were analysed descriptively. To study the differences between groups, the chi-square test, Mann-Whitney U test and Wilcoxon Sign Rank test were used when appropriate. Correlations were analysed by Spearman rank correlation. Comparisons between repeated measures were performed using Friedman's test. Group comparisons were conducted on
HAQ- questions and HAQ-total scores. The observed HAQ score was calculated on 7 domains. The sum of the highest scores in each domain was divided by seven to achieve a total HAQ score. When comparing observed HAQ with reported HAQ, the same 17 questions were used, and a total score calculated. The number of included patients, 51 women and 49 men, was considered adequate, based on a study showing that a change in HAQ of 0.31 points or more over 2 months was needed to ensure a clinically relevant change with a significance level of 5\% and a statistical power of $80 \%$ [24].

All statistical analyses were two-tailed and performed using IBM SPSS version 21(IBM Corp., Armonk, NY, USA). For all tests $p<0.05$ was considered to be significant.

\section{Results}

\section{Demographic and clinical characteristics}

A total of 100 patients, 51 women and 49 men were included in the study. Table 2 shows their characteristics. Both genders had low disease activity, the women were younger, had higher HAQ and had lower grip force than the men. All patients were treated with conventional or biological diseasemodifying anti-rheumatic drugs.

\section{Comparisons between reported and observed HAQ}

Both women and men reported in HAQ1 that their abilities to perform the activities regarding cooking your own food (question 6) and washing and drying yourself (question 12), were better than what was later observed (Table 3).

In addition, women reported their ability to be better than was observed also regarding taking a bath in a bathtub (question 10) and men regarding taking a package of sugar from a shelf (question 13), opening cans with screw cap (question 16) and being able to vacuum (question 18) (Table 3).

In women, the reported HAQ1 score (7 domains) did not significantly differ from the corresponding observed HAQ score, while in men, the reported HAQ1 score was significantly lower than that observed, median (IQR) 0.28 (0.9) versus 0.43 (0.96), $p=0.002$ (Table 3).

Despite the differences in some of the activities, the correlations between reported HAQ1 score (7 domains) and observed HAQ score were strong, $r=0.860, p<$ 0.001 in women, and $r=0.820, p<0.001$ in men.

We then compared the observed HAQ with that reported by the patients after the supervised performance of the different activities, HAQ2. The pattern of differences in the respective activity was very similar to that found when comparing observed HAQ with HAQ1 (Table S1). Reported HAQ2 score (7 domains) and observed HAQ score were in women median (IQR) 0.43 
Table 1 Standardized assessment protocol for Health Assessment Questionnaire response categories "With some difficulty" and "With much difficulty"

\begin{tabular}{|c|c|}
\hline Activity/Question & With some difficulty \\
\hline \multirow[t]{2}{*}{$\begin{array}{l}\text { 3. Are you able to stand up from } \\
\text { a chair without support? }\end{array}$} & $\begin{array}{l}\text { a. With the support of one hand } \\
\text { against the chair }\end{array}$ \\
\hline & b. Succeed on the second attempt \\
\hline \multirow[t]{2}{*}{ 4. Are you able to get in and out of bed? } & a. Uses own technique of raising \\
\hline & b. Succeed on the second attempt \\
\hline \multirow{3}{*}{$\begin{array}{l}\text { 5. Are you able to cut your own meat? } \\
\text { The meat is replaced with half an orange. } \\
\text { Ordinary knife is used }\end{array}$} & a. A little clumsiness \\
\hline & b. Somewhat difficult with the grip \\
\hline & c. Succeed on the second attempt \\
\hline \multirow{3}{*}{$\begin{array}{l}\text { 6. Can you cook your own food? } \\
\text { Patients prepare a soup according to recipe. Normal cooking time } \\
10 \text { min }\end{array}$} & a. A little clumsiness \\
\hline & $\begin{array}{l}\text { b. A bit difficult with grip and handling } \\
\text { of pans and utensils }\end{array}$ \\
\hline & $\begin{array}{l}\text { c. Hard to open a bottle, succeeds on } \\
\text { the second try }\end{array}$ \\
\hline \multirow{2}{*}{$\begin{array}{l}\text { 7. Are you able to lift a full cup or glass } \\
\text { to your mouth? }\end{array}$} & a. A little clumsiness \\
\hline & b. Uses an unusual grip \\
\hline \multirow[t]{2}{*}{ 8. Are you able to walk five steps down a stairway? } & a. Little uncertainty exists \\
\hline & b. Watches carefully when taking a step \\
\hline
\end{tabular}

9. Are you able to walk outdoors on flat ground?

10. Are you able to take a bath? Simulated in bathtub

11. Are you able to get on and off the toilet?

12. Are you able to wash and dry your body? Simulated

13. Are you able to take a $2-k$ bag of sugar of a shelf at head height?

14. Are you able to bend over to pick up clothing from the floor?

\section{With much difficulty}

a. With the support of both hands against the chair

b. Uncertainty / fear exists

c. Succeeds only on the third attempt or more

a. Needs to take support in some way

b. Must use a different technique than usual

c. Succeeds only on the third attempt or more

a. Greater clumsiness, dropping of utensils

b. Uncoordinated cutting

c. Very difficult to grip

d. Succeeds only on the third attempt or more

a. Greater clumsiness, drops the utensils

b. Difficult to open, to lift, must exert oneself

c. Succeeds first on third attempt or more

a. Overflows

b. Uses both hands

a. Holds on to the railing

b. Takes one step at a time

c. Is very uncertain/afraid

a. Very unstable while walking

b. A little unsteady

c. Walks with caution

b. Not able to walk more than $15-20 \mathrm{~m}$

c. Fear of falling exists

a. Succeeds on the third attempt to take the step over

b. Supporting oneself with both hands on the bathtub edge to get in

c. Very difficult to get up, succeeds on the third attempt

d. Manages to get up on the second attempt

a. A little cautious, looking for some support with one hand

b. Manages to get up on the second attempt

a. A little clumsiness with soap and shampoo

b. Not able to reach everywhere (not back and feet) but almost

a. Takes help of both hands

b. Uses an unusual grip

c. Some effort succeeds on the second attempt

a. Some effort

b. Little clumsiness

c. Succeed on the second attempt d. Very uncertain/afraid

a. Takes support with both hands

b. Sits uncontrollably

c. Manages to get up on the third attempt

a. Does not reach multiple body parts, $>2$

b. Very difficult with the balance when drying

c. Needs to sit

a. Risk of dropping the bag due to weak grip

b. Succeeds on the third attempt or more
a. Losing the grip
b. Risk of fall
c. Succeeds on the third attempt or more 
Table 1 Standardized assessment protocol for Health Assessment Questionnaire response categories "With some difficulty" and "With much difficulty" (Continued)

\begin{tabular}{|c|c|c|}
\hline Activity/Question & With some difficulty & With much difficulty \\
\hline \multirow[t]{2}{*}{ 15. Are you able to open car doors? } & $\begin{array}{l}\text { a. Difficulty with the grip, losing the grip, } \\
\text { succeeding on the second attempt }\end{array}$ & a. Very heavy, using both hands \\
\hline & b. Little difficult, it's heavy & b. Succeeds on the third attempt \\
\hline \multirow[t]{3}{*}{ 16. Are you able to open previously opened jars? } & $\begin{array}{l}\text { a. Not directly, succeeds on the second } \\
\text { attempt }\end{array}$ & a. Greater clumsiness \\
\hline & b. Little clumsiness & b. Uses both hands \\
\hline & $\begin{array}{l}\text { c. Takes an unusual technique (for example } \\
\text { holding the jar against the body) }\end{array}$ & c. Risk of dropping the jar due to the grip \\
\hline \multirow[t]{3}{*}{ 17. Are you able to turn a water tap on and off? } & a. Uses an unusual grip & a. Uses both hands \\
\hline & b. Losing the grip & b. Succeeds on the third attempt or more \\
\hline & c. Succeeds on the second attempt & \\
\hline \multirow{4}{*}{$\begin{array}{l}\text { 18. Are you able to vacuum? } \\
\text { Tested on delimited surface, same } \\
\text { for all patients }\end{array}$} & a. Weak grip & a. Loses the grip \\
\hline & $\begin{array}{l}\text { b. Somewhat difficult to pull the vacuum } \\
\text { cleaner }\end{array}$ & b. Very difficult to pull the vacuum cleaner \\
\hline & c. Not able to reach under low furniture & c. Takes short breaks \\
\hline & $\begin{array}{l}\text { d. Uses an unusual technique (e.g. hose } \\
\text { around waist) }\end{array}$ & $\begin{array}{l}\text { d. Not able to reach everywhere on free } \\
\text { surfaces }\end{array}$ \\
\hline \multirow[t]{5}{*}{ 20. Are you able to get in and out of a car? } & a. Uses an unusual technique & a. Sits uncontrollably \\
\hline & b. Some risk of hitting the head & b. Losing balance \\
\hline & c. Succeeds on the second attempt & c. Risk of falling \\
\hline & & d. Great risk of hitting the head \\
\hline & & $\begin{array}{l}\text { e. Succeeds on the third attempt or more } \\
\text { when raised }\end{array}$ \\
\hline
\end{tabular}

Table 2 Characteristics of the patients split by gender

\begin{tabular}{llll}
\hline & $\begin{array}{l}\text { Women } \\
\boldsymbol{n}=\mathbf{5 1}\end{array}$ & $\begin{array}{l}\text { Men } \\
\boldsymbol{n}=\mathbf{4 9}\end{array}$ & $\boldsymbol{p}$-value \\
\hline Age (years) & $58(20)$ & $66(14)$ & 0.008 \\
Disease duration (years) & $5(8)$ & $5(9)$ & 0.590 \\
RF positive, n (\%) & $44(86)$ & $34(69)$ & 0.042 \\
DAS28 & $2.65(1.31)$ & $2.52(1.55)$ & 0.450 \\
HAQ1 & $0.50(0.88)$ & $0.25(0.84)$ & 0.038 \\
Grip force right, N & $117(89)$ & $241(158)$ & $<0.001$ \\
Grip force left, N & $104(105)$ & $245(131)$ & $<0.001$ \\
Grip force mean right + left, N & $126(84)$ & $238(146)$ & $<0.001$ \\
SOFI hands & $1.0(2.0)$ & $1.0(3.0)$ & 0.663 \\
SOFI arms & $0(1.0)$ & $0(2.0)$ & 0.303 \\
SOFI legs & $1.9(3.0)$ & $1.3(2.7)$ & 0.283 \\
SOFI index & $0(3.0)$ & $0(2.0)$ & 0.277 \\
CDMARDs, $n$ (\%) & $24(47)$ & $31(63)$ & 0.103 \\
bDMARDs, n (\%) & $27(53)$ & $18(37)$ & \\
\hline
\end{tabular}

Values are median (IQR), or numbers (\%); $p$-values are differences between women and men

$R F$ rheumatoid factor, $n$ numbers, DAS28 disease activity score calculated on 28 joints, HAQ1 Health Assessment Questionnaire reported at baseline, $N$ Newton, SOFI Signals of Functional Impairment, CDMARDs conventional disease-modifying anti-rheumatic drugs, $6 D M A R D s$ biological disease-modifying anti-rheumatic drugs
(0.96) and $0.57(0.86)$, respectively, $p=0.002$, and in men $0.21(0.57)$ and $0.43(0.96), p<0.001$. The correlations between reported HAQ2 score (7 domains) and observed HAQ score was still strong, $r=0.961, p<0.001$ in women, and $r=0.942, p<0.001$, in men.

\section{Observed HAQ in women and men}

When comparing how women and men performed the observed HAQ activities, it was found that in only two of the 17 items women performed worse than men, namely the question about rising from a chair (question 5), median (IQR) 0.0 (1.0) vs 0.0 (1.0), $p=0.023$ and reaching (number 13), 1.0 (1.0) vs 0.0 (1.0), $p=0.014$. The observed HAQ score (7 domains) did not differ between genders, median (IQR) $0.57(0.9)$ versus 0.43 (0.96), $p=0.292$.

\section{Repeated HAQ measurements}

We further analysed if the reported HAQ was influenced by the patient's experience of the observed HAQ performance by comparisons of HAQ1, HAQ2 and HAQ3 by Friedman's test. For most activities the repeated measurements did not differ significantly (Table S2). 
Table 3 Reported and observed HAQ in women and men. The scores for the 17 questions in reported HAQ1 and observed HAQ are compared, as well as the total HAQ scores for all 7 domains

\begin{tabular}{|c|c|c|c|c|c|c|}
\hline & \multicolumn{3}{|l|}{ Women } & \multicolumn{3}{|l|}{ Men } \\
\hline & HAQ1 reported & HAQ observed & $p$-value & HAQ1 reported & HAQ observed & $p$-value \\
\hline \multicolumn{7}{|l|}{ Rising } \\
\hline HAQ_3 & $0.0(0.8)$ & $0.0(0.0)$ & 0.096 & $0.0(1.0)$ & $0.0(1.0)$ & 0.180 \\
\hline HAQ_4 & $0.0(0.0)$ & $0.0(1.0)$ & 0.180 & $0.0(1.0)$ & $0.0(0.0)$ & 0.157 \\
\hline \multicolumn{7}{|l|}{ Eating } \\
\hline HAQ_5 & $0.0(1.0)$ & $0.0(1.0)$ & 0.248 & $0.0(0.0)$ & $0.0(1.0)$ & 0.058 \\
\hline HAQ_6 & $0.0(0.8)$ & $0.0(1.0)$ & 0.003 & $0.0(0.0)$ & $0.0(1.0)$ & 0.013 \\
\hline HAQ_7 & $0.0(0.0)$ & $0.0(0.0)$ & 0.180 & $0.0(0.0)$ & $0.0(0.0)$ & 1.00 \\
\hline \multicolumn{7}{|l|}{ Walking } \\
\hline HAQ_8 & $0.0(1.0)$ & $0.0(1.0)$ & 0.206 & $0.0(0.0)$ & $0.0(0.8)$ & 0.052 \\
\hline HAQ_9 & $0.0(1.0)$ & $0.0(1.0)$ & 1.00 & $0.0(0.0))$ & $0.0(1.0)$ & 0.071 \\
\hline \multicolumn{7}{|l|}{ Hygiene } \\
\hline HAQ_10 & $0.0(1.0)$ & $0.0(2.0)$ & 0.015 & $0.0(1.0)$ & $0.5(1.8)$ & 0.058 \\
\hline HAQ_11 & $0.0(0.0)$ & $0.0(0.0)$ & 0.317 & $0.0(0.0)$ & $0.0(1.0)$ & 0.317 \\
\hline HAQ_12 & $0.0(1.0)$ & $0.0(1.0)$ & 0.029 & $0.0(1.0)$ & $1.0(1.0)$ & $<0.001$ \\
\hline \multicolumn{7}{|l|}{ Reaching } \\
\hline HAQ_13 & $1.0(1.0)$ & $1.0(1.0)$ & 1.00 & $0.0(1.0)$ & $0.0(1.0)$ & 0.035 \\
\hline HAQ_14 & $0.0(0.0)$ & $0.0(0.5)$ & 1.00 & $0.0(1.0)$ & $0.0(1.0)$ & 0.180 \\
\hline \multicolumn{7}{|l|}{ Gripping } \\
\hline HAQ_15 & $0.0(0.0)$ & $0.0(0.0)$ & 0.564 & $0.0(0.0)$ & $0.0(0.0)$ & 0.317 \\
\hline HAQ_16 & $0.0(1.0)$ & $0.0(1.0)$ & 0.071 & $0.0(1.0)$ & $0.0(1.0)$ & 0.046 \\
\hline HAQ_17 & $0.0(1.0)$ & $0.0(0.5)$ & 0.180 & $0.0(0.0)$ & $0.0(0.0)$ & 0.157 \\
\hline \multicolumn{7}{|l|}{ Other activities } \\
\hline HAQ_18 & $0.0(1.0)$ & $0.0(1.0)$ & 0.096 & $0.0(0.0)$ & $0.0(1.0)$ & 0.002 \\
\hline HAQ_20 & $0.0(1.0)$ & $0.0(1.0)$ & 0.366 & $0.0(1.0)$ & $0.0(1.0)$ & 0.564 \\
\hline \multicolumn{7}{|l|}{ All 7 domains } \\
\hline HAQ_score & $0.50(1.0)$ & $0.57(0.9)$ & 0.325 & $0.28(0.9)$ & $0.43(0.96)$ & 0.002 \\
\hline
\end{tabular}

Values are median (IQR); $p$-values are differences between reported and observed HAQ in respective gender. Bold $p$-values are significant

$H A Q$ Health Assessment Questionnaire

Reported and observed HAQ in relation to grip force

Men had overall significantly higher grip force than women (Table 2).

As shown in Table 4, there was a significant negative correlation between grip force and most of the separate activities in HAQ2 in both women and men. Overall, the correlations between reported HAQ2 score and grip force were moderate, $r=-$ $0.681, p<0.001$ in women and $r=-0.416, p<0.003$, in men.

Looking at grip force and observed HAQ we found similar correlations, $r=-0.698, p<0.001$, in women and $r=-0.516, p<0.001$, in men.

\section{Reported and observed HAQ in relation to SOFI}

The three parts of SOFI were similar in women and men (Table 2). The correlations between SOFI and the different questions in reported HAQ2 were calculated separately for women and men (Table 5).

In women, significant correlations were found both between SOFI hands and legs and most questions in HAQ2. SOFI arms were significantly correlated to the domains of grooming, rising, eating, reaching and other activities (questions 2, 3, 7, 10, 13, 14 and 18).

In men, SOFI hands did significantly correlate with only a few HAQ2 activities. Notably, SOFI legs but not SOFI hands correlated well with HAQ 16 and 17, although both questions are almost entirely dependent on hand function. SOFI arms correlated moderately with the questions about dressing, hygiene, reaching and other activities (questions 1, 2, $10,12,14$ and 18). SOFI legs correlated significantly with all items (Table 5). In both women and 
Table 4 Correlations between grip force assessed with GRIPPIT mean right and left hands and the different activities in reported HAQ2, in women and men

\begin{tabular}{lll}
\hline & Women & Men \\
\hline Grip force & & \\
versus HAQ_1 & $-0.510, \boldsymbol{p}<\mathbf{0 . 0 0 1}$ & $-0.362, \boldsymbol{p}=\mathbf{0 . 0 1 1}$ \\
versus HAQ_2 & $-0.451, \boldsymbol{p}=\mathbf{0 . 0 0 1}$ & $-0.344, \boldsymbol{p}=\mathbf{0 . 0 1 5}$ \\
versus HAQ_3 & $-0.472, \boldsymbol{p}<\mathbf{0 . 0 0 1}$ & $-0.340, \boldsymbol{p}=\mathbf{0 . 0 1 7}$ \\
versus HAQ_4 & $-0.400, \boldsymbol{p}=\mathbf{0 . 0 0 4}$ & $-0.331, \boldsymbol{p}=\mathbf{0 . 0 2 0}$ \\
versus HAQ_5 & $-0.596, \boldsymbol{p}<\mathbf{0 . 0 0 1}$ & $-0.337, \boldsymbol{p}=\mathbf{0 . 0 1 8}$ \\
versus HAQ_6 & $-0.567, \boldsymbol{p}<\mathbf{0 . 0 0 1}$ & $-0.414, \boldsymbol{p}=\mathbf{0 . 0 0 3}$ \\
versus HAQ_7 & $-0.356, \boldsymbol{p}=\mathbf{0 . 0 1 0}$ & $-0.277, \boldsymbol{p}=0.054$ \\
versus HAQ_8 & $-0.216, \boldsymbol{p}=0.129$ & $-0.366, \boldsymbol{p}=\mathbf{0 . 0 1 0}$ \\
versus HAQ_9 & $-0.194, \boldsymbol{p}=0.172$ & $-0.349, \boldsymbol{p}=\mathbf{0 . 0 1 4}$ \\
versus HAQ_10 & $-0.569, \boldsymbol{p}<\mathbf{0 . 0 0 1}$ & $-0.414, \boldsymbol{p}=\mathbf{0 . 0 0 3}$ \\
versus HAQ_11 & $-0.465, \boldsymbol{p}=\mathbf{0 . 0 0 1}$ & $-0.218, \boldsymbol{p}=0.133$ \\
versus HAQ_12 & $-0.489, \boldsymbol{p}<\mathbf{0 . 0 0 1}$ & $-0.365, \boldsymbol{p}=\mathbf{0 . 0 1 0}$ \\
versus HAQ_13 & $-0.582, \boldsymbol{p}<\mathbf{0 . 0 0 1}$ & $-0.379, \boldsymbol{p}=\mathbf{0 . 0 0 7}$ \\
versus HAQ_14 & $-0.569, \boldsymbol{p}<\mathbf{0 . 0 0 1}$ & $-0.371, \boldsymbol{p}=\mathbf{0 . 0 0 9}$ \\
versus HAQ_15 & $-0.516, \boldsymbol{p}<\mathbf{0 . 0 0 1}$ & $-0.414, \boldsymbol{p}=\mathbf{0 . 0 0 3}$ \\
versus HAQ_16 & $-0.454, \boldsymbol{p}=\mathbf{0 . 0 0 1}$ & $-0.364, \boldsymbol{p}=\mathbf{0 . 0 1 0}$ \\
versus HAQ_17 & $-0.397, \boldsymbol{p}=\mathbf{0 . 0 0 4}$ & $-0.348, \boldsymbol{p}=\mathbf{0 . 0 1 4}$ \\
versus HAQ_18 & $-0.585, \boldsymbol{p}<\mathbf{0 . 0 0 1}$ & $-0.367, \boldsymbol{p}=\mathbf{0 . 0 1 0}$ \\
versus HAQ_19 & $-0.560, \boldsymbol{p}<\mathbf{0 . 0 0 1}$ & $-0.381, \boldsymbol{p}=\mathbf{0 . 0 0 7}$ \\
versus HAQ_20 & $-0.466, \boldsymbol{p}=\mathbf{0 . 0 0 1}$ & $-0.376, \boldsymbol{p}=\mathbf{0 . 0 0 8}$ \\
versus total HAQ2_score & $-0.681, \boldsymbol{p}<\mathbf{0 . 0 0 1}$ & $-0.416, \boldsymbol{p}=\mathbf{0 . 0 0 3}$ \\
\hline Bold 0 -valn & &
\end{tabular}

Bold $p$-values are significant

$H A Q$ Health Assessment Questionnaire

men, the associations of observed HAQ with SOFI components were similar to those of HAQ2 with SOFI components.

Overall, the correlations between reported HAQ2 total score and SOFI index were moderate, $r=0.721, p<0.001$ in women, and $r=0.634, p<0.001$ in men. Similar moderate correlations were found between HAQ observed total score and SOFI index, $r=0.762, p<0.001$ in women and $r=0.693, p<0.001$ in men.

\section{Discussion}

This study shows that women and men with established RA and low disease activity reported their activity limitations, assessed by the self-administered HAQ, in relatively good agreement with that noticed by an independent observer. However, in some activities the women as well as the men reported their abilities to be better than those observed, both before the observed performance and in the subsequent reported questionnaires. In both genders there was a negative correlation between grip force and most of the separate activities in HAQ. Furthermore, the functional impairment assessed by SOFI showed in women correlations especially between SOFI hands and legs and HAQ and in men mainly between SOFI legs and HAQ.

The HAQ disability index, specifically designed for arthritic patients, was among the first instruments to assess the patient's own opinion of her/his health status. The Swedish version of HAQ, presented in 1988, showed a high degree of reliability and validity in patients with RA [20]. Neither in the original HAQ from Stanford [25] nor in the Swedish version [20], the validity was tested separately in women and men, but in these studies as well as in the present study, a strong correlation was found between the patient's selfadministered abilities and the observer's opinion. In the US patients the correlation was $r=0.88$ and in the Swedish patients $r=0.71$. Of interest, in the latter report the authors suggested that men may tend to underreport daily life difficulties [20].

The agreement between patient reported HAQ and observed HAQ was, however, not true for all activities. As discrepancies were present also in the reported HAQ after the practical performance of the activities, we consider that the patient's opinion was not dependent on unawareness of his/her own ability. Instead, the discrepancies may relate to divergent perceptions of disability. When, in the present study, the reported and observed activities differed, the patient's assessment score was always lower than that observed, in both genders. As the patients relate to their own situation, their lower rating probably reflects that they after several years with RA may have changed their perception of what is difficult. This interpretation agrees with an earlier study [11] comparing the reported HAQ of 51 patients with that observed, in which the average difference was low, mean (SD) 0.09 (0.39), but wide in range and without any significant gender difference. In that study the patients' over-reported functional ability was associated with longer disease duration, why they may have been more inclined to adapt to the loss of functional ability.

Due to the subjective nature of the observed HAQ, aspects of trustworthiness were considered regarding the data collection procedure [26]. The standardized protocol was developed and used to ensure consistent assessments based on the same criteria, and the different levels of difficulty were tested before they were applied to this study. To eliminate sources of bias, the time factor was not considered as an assessment criterion. Thus, the standardized observed assessment procedure estimated the functional performance regardless of the time factor. In addition, the patients did not receive any feedback from the observer on how their performances were graded in order not to affect the participants' own subsequent assessments. To further avoid bias the observer was blinded for the result of the reported HAQ. 
Table 5 Correlations between SOFI hands, arms and legs and the different activities in reported HAQ2, in women and men

\begin{tabular}{|c|c|c|c|c|c|c|}
\hline & Women & & & Men & & \\
\hline & SOFI hands & SOFI arms & SOFI legs & SOFI hands & SOFI arms & SOFI legs \\
\hline HAQ_1 & $0.208, p=0.144$ & $0.097, p=0.500$ & $0.439, p=\mathbf{0 . 0 0 1}$ & $0.076, p=0.603$ & $0.381, p=\mathbf{0 . 0 0 7}$ & $0.607, \boldsymbol{p}<\mathbf{0 . 0 0 1}$ \\
\hline HAQ_2 & $0.463, \boldsymbol{p}=\mathbf{0 . 0 0 1}$ & $0.313, p=\mathbf{0 . 0 2 6}$ & $0.349, \boldsymbol{p}=\mathbf{0 . 0 1 2}$ & $0.321, \boldsymbol{p}=\mathbf{0 . 0 2 5}$ & $0.341, p=\mathbf{0 . 0 1 7}$ & $0.627, \boldsymbol{p}<\mathbf{0 . 0 0 1}$ \\
\hline HAQ_3 & $0.291, \boldsymbol{p}=\mathbf{0 . 0 3 8}$ & $0.330, p=\mathbf{0 . 0 1 8}$ & $0.512, \boldsymbol{p}<\mathbf{0 . 0 0 1}$ & $0.161, p=0.268$ & $0.226, p=0.118$ & $0.652, \boldsymbol{p}<\mathbf{0 . 0 0 1}$ \\
\hline HAQ_4 & $0.313, \boldsymbol{p}=\mathbf{0 . 0 2 6}$ & $0.183 p=0.198$ & $0.429, \boldsymbol{p}=\mathbf{0 . 0 0 2}$ & $0.141, p=0.333$ & $0.248, p=0.086$ & $0.460, \boldsymbol{p}=\mathbf{0 . 0 0 1}$ \\
\hline HAQ_5 & $0.490, \boldsymbol{p}<\mathbf{0 . 0 0 1}$ & $0.175, p=0.221$ & $0.385, \boldsymbol{p}=\mathbf{0 . 0 0 5}$ & $0.089, p=0.545$ & $0.271, p=0.060$ & $0.546, \boldsymbol{p}<\mathbf{0 . 0 0 1}$ \\
\hline HAQ_6 & $0.447, \boldsymbol{p}=\mathbf{0 . 0 0 1}$ & $0.051, p=0.722$ & $0.485, \boldsymbol{p}<\mathbf{0 . 0 0 1}$ & $0.035, p=0.813$ & $0.111, p=0.449$ & $0.316, \boldsymbol{p}=\mathbf{0 . 0 2 9}$ \\
\hline HAQ_7 & $0.630, \boldsymbol{p}<\mathbf{0 . 0 0 1}$ & $0.301, p=\mathbf{0 . 0 3 2}$ & $0.397, p=\mathbf{0 . 0 0 4}$ & $0.252, p=0.081$ & $0.054, p=0.713$ & $0.417, \boldsymbol{p}=\mathbf{0 . 0 0 3}$ \\
\hline HAQ_8 & $0.320, \boldsymbol{p}=\mathbf{0 . 0 2 2}$ & $0.186, p=0.190$ & $0.643, \boldsymbol{p}<\mathbf{0 . 0 0 1}$ & $0.191, p=0.189$ & $0.248, p=0.086$ & $0.483, \boldsymbol{p}=\mathbf{0 . 0 0 1}$ \\
\hline HAQ_9 & $0.319, \boldsymbol{p}=\mathbf{0 . 0 2 3}$ & $0.094, p=0.513$ & $0.399, p=\mathbf{0 . 0 0 4}$ & $0.030, p=0.838$ & $0.224, p=0.122$ & $0.579, \boldsymbol{p}<\mathbf{0 . 0 0 1}$ \\
\hline HAQ_10 & $0.483, \boldsymbol{p}<\mathbf{0 . 0 0 1}$ & $0.364, \boldsymbol{p}=\mathbf{0 . 0 0 9}$ & $0.454, \boldsymbol{p}=\mathbf{0 . 0 0 1}$ & $0.370, p=\mathbf{0 . 0 0 9}$ & $0.419, \boldsymbol{p}=\mathbf{0 . 0 0 3}$ & $0.597, \boldsymbol{p}<\mathbf{0 . 0 0 1}$ \\
\hline HAQ_11 & $0.448, \boldsymbol{p}=\mathbf{0 . 0 0 1}$ & $0.271, p=0.054$ & $0.365, p=\mathbf{0 . 0 0 8}$ & $0.054, p=0.712$ & $0.209, p=0.152$ & $0.531, \boldsymbol{p}<\mathbf{0 . 0 0 1}$ \\
\hline HAQ_12 & $0.458, \boldsymbol{p}=\mathbf{0 . 0 0 1}$ & $0.153, p=0.284$ & $0.318, \boldsymbol{p}=\mathbf{0 . 0 2 3}$ & $0.275, p=0.056$ & $0.326, \boldsymbol{p}=\mathbf{0 . 0 2 2}$ & $0.531, \boldsymbol{p}<\mathbf{0 . 0 0 1}$ \\
\hline HAQ_13 & $0.498, \boldsymbol{p}<\mathbf{0 . 0 0 1}$ & $0.302, p=\mathbf{0 . 0 3 1}$ & $0.324, \boldsymbol{p}=\mathbf{0 . 0 2 0}$ & $0.223, p=0.123$ & $0.143, p=0.328$ & $0.356, \boldsymbol{p}=\mathbf{0 . 0 1 3}$ \\
\hline HAQ_14 & $0.585, \boldsymbol{p}<\mathbf{0 . 0 0 1}$ & $0.431, \boldsymbol{p}=\mathbf{0 . 0 0 2}$ & $0.510, p<0.001$ & $0.174, p=0.240$ & $0.305, \boldsymbol{p}=\mathbf{0 . 0 3 3}$ & $0.532, \boldsymbol{p}<\mathbf{0 . 0 0 1}$ \\
\hline HAQ_15 & $0.339, \boldsymbol{p}=\mathbf{0 . 0 1 5}$ & $0.193, p=0.175$ & $0.384, p=0.005$ & $0.237, p=0.101$ & $0.070, p=0.635$ & $0.349, \boldsymbol{p}=\mathbf{0 . 0 1 5}$ \\
\hline HAQ_16 & $0.463, \boldsymbol{p}<\mathbf{0 . 0 0 1}$ & $0.090, p=0.530$ & $0.241, p=0.089$ & $0.145, p=0.319$ & $0.067, p=0.648$ & $0.464, \boldsymbol{p}=\mathbf{0 . 0 0 1}$ \\
\hline HAQ_17 & $0.528, \boldsymbol{p}<\mathbf{0 . 0 0 1}$ & $0.078, p=0.584$ & $0.384, p=0.005$ & $0.189, p=0.194$ & $0.169, p=0.246$ & $0.525, \boldsymbol{p}<\mathbf{0 . 0 0 1}$ \\
\hline HAQ_18 & $0.368, \boldsymbol{p}=\mathbf{0 . 0 0 8}$ & $0.362, \boldsymbol{p}=\mathbf{0 . 0 0 9}$ & $0.505, \boldsymbol{p}<\mathbf{0 . 0 0 1}$ & $0.134, p=0.358$ & $0.340, \boldsymbol{p}=\mathbf{0 . 0 1 7}$ & $0.505, \boldsymbol{p}<\mathbf{0 . 0 0 1}$ \\
\hline HAQ_19 & $0.459, \boldsymbol{p}=\mathbf{0 . 0 0 1}$ & $0.232, p=0.099$ & $0.363, p=0.009$ & $0.035, p=0.813$ & $0.227, p=0.177$ & $0.434, \boldsymbol{p}=\mathbf{0 . 0 0 2}$ \\
\hline HAQ_20 & $0.434, \boldsymbol{p}=\mathbf{0 . 0 0 1}$ & $0.251, p=0.076$ & $0.579, \boldsymbol{p}<\mathbf{0 . 0 0 1}$ & $0.238, p=0.099$ & $0.119, p=0.417$ & $0.568, \boldsymbol{p}<\mathbf{0 . 0 0 1}$ \\
\hline HAQ_score & $0.584, \boldsymbol{p}<\mathbf{0 . 0 0 1}$ & $0.316, \boldsymbol{p}=\mathbf{0 . 0 2 4}$ & $0.614, \boldsymbol{p}<\mathbf{0 . 0 0 1}$ & $0.367, p=\mathbf{0 . 0 0 9}$ & $0.354, \boldsymbol{p}=\mathbf{0 . 0 1 3}$ & $0.728, \boldsymbol{p}<\mathbf{0 . 0 0 1}$ \\
\hline
\end{tabular}

Bold $p$-values are significant

SOFI Signals of Functional Impairment, HAQ Health Assessment Questionnaire

As HAQ reflects patient perceived abilities in performance limited to certain areas of daily life, many related to housework, one might suspect that men are not always aware of their abilities. However, this suspicion could not be verified here. Neither did the questions deal with rare activities as there were very few questions, which were not answered by the patients. We are thus confident that HAQ is a reliable instrument to assess activity limitation but realise the wish to add modern lifestyle activities [27], as well as supplements with the patient's opinion about the impact of the disability [28].

Similar to our findings, many studies report higher HAQ scores in women than in men $[2,5,6]$, mostly related to higher disease activity in women. However, in one study the HAQ score did not differ between genders at diagnosis [7]. Interestingly, in a study establishing normative values for HAQ scores in the general population in which the functional disability increased with age, the gender difference disappeared after adjustment for age [29].

Apart from disease activity and joint damage, grip force has been reported to correlate with HAQ score in patients with RA, women as well as men [10]. Also, in the present study we found a negative correlation between grip force and most of the separate activity scores in HAQ in both genders. To note, such a correlation was as well found in a few activities not obviously dependent on the use of hands, i.e. walking (in men). We suggest this to be explained by good muscle strength in hands and legs.

We could verify that the women with RA had significantly lower grip force than the men, with mean values close to those earlier reported after a disease duration of 5 years and lower than those of healthy referents [30]. Despite the discrepancy in grip force, the total HAQ score was not significantly different between genders. This finding resembles the situation in healthy persons, where women and men had similar HAQ score, despite lower grip force in the women [30]. Therefore, only grip force reduced to a certain level seems to have functional importance, which has been shown by grouping RA patients with respect to grip force [10].

Physical impairment, assessed by SOFI, also correlated with different activities in HAQ, but unequally between genders. In women, SOFI hands and legs correlated with most activities, whereas in men SOFI legs had the greatest impact on the HAQ activities. The association between impairment and activity limitation was, though, 
moderate which is in line with earlier reports [31, 32]. It is obvious that the range of movements of individual joints has different implications for the various activities of the $\mathrm{HAQ}$, in agreement with a previous report [14] and that SOFI leg is an important predictor of HAQ [30].

This study has certain limitations. The study includes relatively small numbers of patients and these had relatively well-controlled disease. The interpretation of the present findings is thus restricted to patients with low disease activity and limited disability. It should also be mentioned that since a main aim of this study was to consider possible gender differences in $\mathrm{HAQ}$, sampling was stratified by gender to avoid getting men underrepresented in the study. A strength is that the present study, unlike most earlier studies in this field, was performed during a period when active therapy with modern treatments had been instituted. A further strength is that all observed assessments were performed by the same observer in the same environment.

\section{Conclusions}

In both women and men with RA and low disease activity the correlations between reported and observed HAQ scores were strong. We found no evidence supporting the possibility that the patient's opinion was dependent on unawareness of his/her own ability. Instead, we suggest that when the patients scored their ability to be better than that observed it might reflect that the patients after several years with RA may have changed their opinion about what is difficult. Furthermore, activity limitation was closely related to grip force and to some extent to physical function in both women and men.

\section{Supplementary Information}

The online version contains supplementary material available at https://doi. org/10.1186/s41927-021-00184-5.

Additional file 1 : Table S1. Reported $\mathrm{HAQ} 2$ and observed $\mathrm{HAQ}$ in women and men. The scores for the 17 questions are compared as well as the total HAQ score for all 7 domains. Table S2. Comparison of HAQ1, HAQ2 and HAQ3 in women and men respectively.

\section{Abbreviations \\ bDMARDs: Biological disease-modifying anti-rheumatic drugs; cDMARDs: Conventional disease-modifying anti-rheumatic drugs; DAS28: Disease Activity Score calculated on 28 joints; HAQ: Health Assessment Questionnaire; RA: Rheumatoid arthritis; IQR: Interquartile range; SOFI: Signals Of Functional Impairment}

\section{Acknowledgements}

The authors wish to thank all the participants in the study for their cooperation. We would also like to thank the occupational therapists Marianne Olsson and Jilan Taher for their support and for facilitating the conduct of the study.

\section{Authors' contributions}

Conception and design of the study: VB, KF, BS and $\mathrm{IH}$. Responsibility for the performance of the study and recruitment of patients VB, data acquisition: VB and KF. Statistical analysis: MA. Data interpretation and conclusion analyses: VB, MA, KF, BS and $\mathrm{H}$. Draft and revision of the manuscript $\mathrm{VB}, \mathrm{MA}$, $\mathrm{KF}, \mathrm{BS}$ and $\mathrm{IH}$. All authors have approved the submitted version of the manuscript and have agreed also to be personally accountable for the author's own contributions and to ensure that questions related to the accuracy or integrity of any part of the work are appropriately investigated, resolved, and the resolution documented in the literature.

\section{Funding}

Supported by unrestricted grants from Stiftelsen Thelma Zoégas Fond, the Swedish Rheumatism Association, and the Foundation for Assistance to Disabled People in Skåne (Stiftelsen för Bistånd åt Rörelsehindrade i Skåne). The funders had no direct role in the conduct of the reported study, analysis of the data or writing of the manuscript. Open Access funding provided by Lund University.

\section{Availability of data and materials}

The datasets used are available from the corresponding author on reasonable request.

\section{Ethics approval and consent to participate}

The study was conducted in accordance with the Declaration of Helsinki and was approved by The Regional Ethical Review Board at Lund University, Lund, Sweden (Dnr. 2010/554). All patients were provided with oral and written study information and written consent was obtained.

Consent for publication

Not applicable.

\section{Competing interests}

The authors declare that they have no competing interests.

\section{Author details}

${ }^{1}$ Department of Health Sciences, Faculty of Medicine, Lund University, Lund, Sweden. 'Department of Medicine, Section of Rheumatology, Helsingborg's Hospital, Helsingborgs lasarett, Olympiahuset plan 2, S-251 87 Helsingborg,

Sweden. ${ }^{3}$ Department of Rheumatology, Faculty of Medicine, Lund

University, Lund, Sweden. ${ }^{4}$ Spenshult Research and Development Center, Halmstad, Sweden. ${ }^{5}$ Department of Research and Education, Skånevård Sund, Region Skåne, and Helsingborg's Hospital, Helsingborg, Sweden.

${ }^{6}$ Department of Medicine, Division of Gastroenterology and Rheumatology, Karolinska Institutet, Huddinge, Stockholm, Sweden. 'Rheumatology Unit, Karolinska University Hospital, Stockholm, Sweden.

Received: 14 August 2020 Accepted: 17 February 2021

Published online: 03 May 2021

\section{References}

1. Hallert E, Thyberg I, Hass U, Skargren E, Skogh T. Comparison between women and men with recent onset rheumatoid arthritis of disease activity and functional ability over two years (the TIRA project). Ann Rheum Dis. 2003;62:667-70.

2. Tengstrand B, Ahlmén M, Hafström I. The influence of sex on rheumatoid arthritis: a prospective study of onset and outcome after 2 years. J Rheumatol. 2004;31:214-22.

3. Forslind K, Hafström I, Ahlmén M, Svensson B, for the BARFOT Study Group. Sex: a major predictor of remission in early rheumatoid arthritis? Ann Rheum Dis. 2007;66:46-52.

4. likuni N, Sato E, Hoshi M, Inoue E, Taniguchi A, Hara M, et al. The influence of sex on patients with rheumatoid arthritis in a large observational cohort. J Rheumatol. 2009;36:508-11.

5. Ahlmén M, Svensson B, Albertsson K, Forslind K, Hafström I, BARFOT study group. Influence of gender on assessments of disease activity and function in early rheumatoid arthritis in relation to radiographic joint damage. Ann Rheum Dis. 2010;69:230-3.

6. Hafström I, Bala V, Albertsson K, Forslind K, Svensson B, BARFOT study group. Joint destruction in early rheumatoid arthritis over 8 years is similar 
in women and men despite apparently higher disease activity and poorer function in women. Ann Rheum Dis. 2011;70:709-10.

7. Hallert E, Björk M, Dahlström Ö, Skogh T, Thyberg I. Disease activity and disability in women and men with early rheumatoid arthritis (RA): an 8-year followup of a Swedish early RA project. Arthritis Care Res. 2012;64:1101-7.

8. Andersson MLE, Forslind K, Hafström I, BARFOT study group. Patients with early rheumatoid arthritis in the 2000s have equal disability and pain despite less disease activity compared with the 1990s: data from the BARFOT study over 8 years. J Rheumatol. 2017;44:723-31.

9. Andersson ML, Forslind K, Hafström I. Comparing five year out-come in two cohorts of patients with early rheumatoid arthritis - a BARFOT study. Open Rheumatol J. 2015;9:8-15.

10. Thyberg I, Hass UA, Nordenskiöld U, Gerdle B, Skogh T. Activity limitation in rheumatoid arthritis correlates with reduced grip force regardless of sex: the Swedish TIRA project. Arthritis Rheum. 2005;53:886-96

11. Van den Ende CH, Hazes JM, Le Cessie S, Breedveld FC, Dijkmans BA. Discordance between objective and subjective assessment of functional ability of patients with rheumatoid arthritis. Br J Rheumatol. 1995;34:951-5.

12. Häkkinen A, Kautiainen $H$, Hannonen P, Ylinen J, Mäkinen H, Sokka T. Muscle strength, pain, and disease activity explain individual subdimensions of the Health Assessment Questionnaire disability index, especially in women with rheumatoid arthritis. Ann Rheum Dis. 2006;65:30-4.

13. Sokka T, Kankainen A, Hannonen P. Scores for functional disability in patients with rheumatoid arthritis are correlated at higher levels with pain scores than with radiographic scores. Arthritis Rheum. 2000;43:386-9.

14. Häkkinen A, Kautiainen H, Hannonen P, Ylinen J, Arkela-Kautiainen M, Sokka T. Pain and joint mobility explain individual subdimensions of the Health Assessment Questionnaire (HAQ) disability index in patients with rheumatoid arthritis. Ann Rheum Dis. 2005;64:59-63.

15. Bilberg A, Bremell T, Bjersing J, Mannerkorpi K. High prevalence of widespread pain in women with early rheumatoid arthritis. Scand J Rheumatol. 2018:47:447-54.

16. Lindqvist E, Saxne T, Geborek P, Eberhardt K. Ten year outcome in a cohort of patients with early rheumatoid arthritis: health status, disease process, and damage. Ann Rheum Dis. 2002;61:1055-9.

17. Lee KE, Choi SE, Xu H, Kang JH, Park DJ, Lee SS. HAQ score is an independent predictor of sustained remission in patients with rheumatoid arthritis. Rheumatol Int. 2017;37:2027-34.

18. Thyberg I, Dahlström Ö, Björk M, Arvidsson P, Thyberg M. Potential of the $\mathrm{HAQ}$ score as clinical indicator suggesting comprehensive multidisciplinary assessments: the Swedish TIRA cohort 8 years after diagnosis of RA. Clin Rheumatol. 2012;31:775-83.

19. Arnett FC, Edworthy SM, Bloch DA, McShane DJ, Fries JF, Cooper NS, et al. The American Rheumatism Association 1987 revised criteria for the classification of rheumatoid arthritis. Arthritis Rheum. 1988;31:315-24.

20. Ekdahl C, Eberhardt K, Andersson SI, Svensson B. Assessing disability in patients with rheumatoid arthritis. Scand J Rheumatol. 1988;17:263-71.

21. Prevoo ML, van't Hof MA, Kuper $H H$, van Leeuwen MA, van de Putte $L B$, van Riel PL. Modified disease activity scores that include twenty-eight-joint counts. Development and validation in a prospective longitudinal study of patients with rheumatoid arthritis. Arthritis Rheum. 1995;38:44-8.

22. Nordenskiöld UM, Grimby G. Grip force in patients with rheumatoid arthritis and fibromyalgia and in healthy subjects. A study with the Grippit instrument. Scand J Rheumatol. 1993;22:14-9.

23. Eberhardt KB, Svensson B, Moritz U. Functional assessment of early rheumatoid arthritis. Br J Rheumatol. 1988;27:364-71.

24. Greenwood MC, Doyle DV, Ensor M. Does the Stanford Health Assessment Questionnaire have potential as a monitoring tool for subjects with rheumatoid arthritis? Ann Rheum Dis. 2001;60:344-8.

25. Fries JF, Spitz P, Kraines RG, Holman HR. Measurement of patient outcome in arthritis. Arthritis Rheum. 1980;23:137-45.

26. Lincoln YS, Guba EG. Naturalistic inquiry. Newbury Park: SAGE Publications; 1985.

27. Bukhari M, Kent A. How rheumatologists assess disability in the current era needs an overhaul: focus on the Health Assessment Questionnaire. Rheumatology (Oxford). 2020;59:267-8.

28. Hewlett S, Smith AP, Kirwan JR. Values for function in rheumatoid arthritis: patients, professionals, and public. Ann Rheum Dis. 2001;60:928-33.

29. Krishnan E, Sokka T, Häkkinen A, Hubert H, Hannonen P. Normative values for the Health Assessment Questionnaire disability index: benchmarking disability in the general population. Arthritis Rheum. 2004;50:953-60.
30. Björk MA, Thyberg IS, Skogh T, Gerdle BU. Hand function and activity limitation according to health assessment questionnaire in patients with rheumatoid arthritis and healthy referents: 5-year followup of predictors of activity limitation (The Swedish TIRA Project). J Rheumatol. 2007:34:296-302.

31. Badley EM, Wagstaff $S$, Wood PH. Measures of functional ability (disability) in arthritis in relation to impairment of range of joint movement. Ann Rheum Dis. 1984:43:563-9.

32. Eberhardt $\mathrm{KB}$, Fex E. Functional impairment and disability in early rheumatoid arthritis-development over 5 years. J Rheumatol. 1995;22:103742.

\section{Publisher's Note}

Springer Nature remains neutral with regard to jurisdictional claims in published maps and institutional affiliations.
Ready to submit your research? Choose BMC and benefit from:

- fast, convenient online submission

- thorough peer review by experienced researchers in your field

- rapid publication on acceptance

- support for research data, including large and complex data types

- gold Open Access which fosters wider collaboration and increased citations

- maximum visibility for your research: over $100 \mathrm{M}$ website views per year

At BMC, research is always in progress.

Learn more biomedcentral.com/submissions 